\title{
Age differences in place-of-articulation phoneme boundary
}

\author{
WILLY DE WEIRDT \\ University of Ghent, Ghent, Belgium
}

\begin{abstract}
Three 10-step [pa]-[to] continua were constructed with F2 as the single place-of-articulation cue. The continua were different in F3, which was rising, level, or falling. Identification tests using these continua were presented to 22 first-, second-, and fourth-grade children. Using logit analysis, individual phoneme boundary values were calculated for each F3 level. It was found that the phoneme-boundary shifts to slightly higher F2 starting values as age increases. Although no definite explanation is available yet, some possible interpretations are discussed.
\end{abstract}

In De Weirdt (1985), age differences in a phoneme boundary were reported. First-grade children (tested at ages 6:2 and 6:10), and third- and fourth-grade children (mean age 9:5) and adults took an identification test with three-formant stimuli on a [po]-[tə] place-of-articulation continuum. Stimuli varied in F2-transition only. With increasing age, more stimuli were heard as [po]. The phoneme boundary shifted to slightly, but significantly, higher F2 starting values $(1414 \mathrm{~Hz}$ at age $6: 2,1454 \mathrm{~Hz}$ at age $6: 10,1484 \mathrm{~Hz}$ at age 9:5, and $1506 \mathrm{~Hz}$ for 18 -year-olds).

This finding is not completely isolated. Simon and Fourcin (1978) found small phoneme-boundary changes in the labeling of voice onset time (VOT) continua with children ranging from 2 to 14 years, but thought it might have been a data analysis artefact. Elliott, Longinotti, Meyer, Raz, and Zucker (1981) found differences between adaptively estimated [ba]-[da] and [da]-[ga] boundaries of 6year-olds, 10-year-olds, and adults. But the age trend was not always very consistent. Five-formant stimuli, with or without burst and varying in F2 and F3, were used. For continua with bursts, 6-year-olds' [ba]-[da] and [da]-[ga] boundaries were at lower F2/F3 values than those of 10year-olds and adults. With burstless stimuli, only [ba][da] boundary differences showed up. For 10-year-olds boundaries occurred at lower F2/F3 values than they did for both 6-year-olds and adults.

Our previous [pə]-[to] results showed a more consistent age trend, but the phoneme-boundary change may have been due to a stimulus peculiarity. In all stimuli of the continuum, F3 fell slightly (from 2534 to $2275 \mathrm{~Hz}$ ). So F3 always points to an alveolar sound, such as [t].

The author is "aangesteld navorser" of the Belgian Nationaal Fonds voor Wetenschappelijk Onderzoek. Thanks are extended to $M$. Vanwormhoudt, J. P. Martens, and B. Van Coile of the Laboratory for Electronics and Metrology, for making available the equipment and some software for synthesizing the stimuli, and to G. De Soete and an anonymous reviewer for their helpful advice on an earlier draft of this paper. The author's mailing address is: Dienst voor Psychologie, Rijksuniversiteit Gent, H. Dunantlaan 2, B9000-Gent, Belgium.
Maybe younger children are more influenced by the F3 cue-or less influenced by F2-than older ones. One might even speculate that speech categorization in younger children is generally based more on the higher frequency information in the acoustic signal. We were led to this presumption by the results of Irwin, Ball, Kay, Stillman, and Rosser (1985), who demonstrated that auditory temporal acuity develops differently in different frequency regions. They measured the minimum detectable gap in octave-band noises centered around 500,1000, and $2000 \mathrm{~Hz}$ at various ages between 6 and 23 years. Especially between the ages of 6 and 10 , the minimum detectable gap decreased, but did so substantially only with the 500-Hz stimulus. Irwin et al. (1985, see Figure 2) did not cover the whole frequency range, but their data suggest that a general trend may exist. The difference between the level of temporal acuity at age 6 and that of the adult increases as one descends the frequency scale. This pattern might also apply to formants in speech sounds. Assuming that temporal processing aspects are probably crucial for the perceptual effectiveness of formant transition cues, the deviating [po]-[to] phoneme boundaries of younger children may be due to poorer temporal resolution of the (lower frequency) F2 cue, resulting in higher "weights" for F3.

It would be interesting to determine if the F3 cue is more important for younger children. If this were true, however, the developmental shift of the phoneme boundary would be only a stimulus artefact. Accordingly, the earlier study (De Weirdt, 1985) was replicated with stimulus continua at three F3-levels: falling, level, and rising. If younger subjects are more sensitive to the F3 cue, different results would be expected for these three F3 levels. With falling F3 (e.g., cuing an alveolar sound such as $t$ ), younger subjects would hear more tos, and have lower F2 phoneme boundaries. With rising F3 (pointing to labial sounds such as [p], younger subjects would hear more [pe]s and have higher $\mathrm{F} 2$ phoneme boundaries. With level F3, no shifting of the phoneme boundary would be predicted. 


\section{METHOD}

Stimuli

Thirty three-formant stimuli were generated using a cascade-model synthesis program (Van Coile, 1985) on a laboratory-built minicomputer. The stimuli varied in the second and third formants and were $130 \mathrm{msec}$ long. After $50 \mathrm{msec}$ of formant transitions, $80 \mathrm{msec}$ of the steady state values for the Dutch schwa [a] were implemented: F1 at $465 \mathrm{~Hz}, \mathrm{~F} 2$ at $1475 \mathrm{~Hz}$, and F3 at $2275 \mathrm{~Hz}$ (Govaerts, 1978). Formant transitions were not linear, but were negatively accelerated. F1 started at $190 \mathrm{~Hz}$. There were $10 \mathrm{~F} 2$ starting values, ranging from $1144 \mathrm{~Hz}$ (extreme [pə]) to $1720 \mathrm{~Hz}$ (extreme [tə]), in equal steps of $64 \mathrm{~Hz}$. For all F2 levels, F3 started at $2015 \mathrm{~Hz}$ (rising), $2275 \mathrm{~Hz}$ (level), or $2535 \mathrm{~Hz}$ (falling). The fundamental frequency fell over the entire length of the stimuli from 143 to $87 \mathrm{~Hz}$. During formant transitions, root-mean-square (RMS) power fell to $67 \%$ of the starting value, and, except for a 20 -msec decay, remained constant during the steady state vowel. The present $F 2$ continuum with falling F3 is identical to that used previously (De Weirdt, 1985), except that it was now at one stimulus step $(64 \mathrm{~Hz})$ lower F2 starting values. A second difference was that, in the present stimuli, the RMS power contour fell further during formant transitions (only to about $85 \%$ of the starting value in the stimuli of the previous study).

The 30 stimuli of the three $F 2$ continua were recorded four times in a different random order, on TDK GX tape, using a Revox A700 tape recorder. The stimuli were recorded with a $4.2-\mathrm{sec}$ interstimulus interval.

\section{Subjects}

The subjects were normal Flemish children from first-, second-, and fourth-grade classes. Twenty-four first graders, 23 second graders, and 22 fourth graders were tested to obtain acceptable data for 22 subjects from each grade level. The subjects were selected randomly in two schools, after the elimination of children with a history of hearing disorders and general or specific backwardness. The mean ages were 6,2 (first grade), 7,4 (second grade), and 9,2 (fourth grade). There were about an equal number of boys and girls in each age group.

\section{Procedure}

The subjects were tested in a quiet room at their school. Stimuli were played on a Philips NR $\mathbf{4 0 0}$ tape recorder through Beyer DT 220 headphones at a comfortable listening level of $72 \mathrm{~dB}$ SPL (measured at the steady-state vowel). Before the actual identification test was presented, the extreme stimuli (lowest and highest F2 and F3) were each played five times; these were explicitly announced by the experimenter as being [po] and [to] articulated by a robot that was learning to talk. Next, a criterion test was played, with eight presentations of the same extreme stimuli, in random order. The purpose of the criterion test was to enhance task understanding and labeling confidence, and also to ascertain that each subject's phoneme boundary was well within the stimulus range. Three subjects failed to pass the criterion test with fewer than three errors. Two first-grade children and 1 second-grade child labeled all stimuli as [to], and were excluded from further participation. As in the previous study (De Weirdt, 1985), the subjects who failed the criterion test were perfectly able to identify large series of various natural speech sounds uttered by the experimenter. Thus, criterion failure is clearly associated with the synthetic nature of the stimuli. Three first graders, 4 second graders, and 2 fourth graders made one error on the criterion test. One fourth grader committed two errors. The remaining subjects passed the criterion test without any errors. The subjects were instructed to respond to each stimulus by saying [pa] or [t?].

\section{RESULTS AND DISCUSSION}

For each individual, F2 phoneme-boundary values were calculated for each $\mathrm{F} 3$ condition. ${ }^{1}$ The $50 \%$ crossover point of the best-fitting logistic curve was chosen as the phoneme-boundary estimate (Baker \& Nelder, 1978). An age $\times \mathrm{F} 3$ level repeated measures analysis of variance was performed on these estimates. Cell means are presented in Table 1 . The F3 effect is highly significant $[F(2,126)=84.20, p<.001]$. Lower $\mathrm{F} 3$ values result in lower $\mathrm{F} 2$ phoneme-boundary values (see Table 1). The age effect is also significant $[F(2,63)=4.50, p<.025]$. As in our previous study, the phoneme boundary shifts to higher F2 starting values as the age increases. The F3 $X$ age interaction is not significant $(F<1)$, so the age effect is not confined to stimuli with falling F3. A posteriori pairwise comparisons (HSD) reveal that only first- and fourth-grade rising-F3 phoneme boundaries are significantly different $[q(3,63)=4.17, p<.05]$. With level F3, first-grade values differ significantly from both second- and fourth-grade values [1st- vs. 2nd-grade, $q(3,63)=3.75, p<.05 ; 1$ st- vs. 4th-grade, $q(3,63)=$ $3.80, p<.05]$. Age differences are not significant with falling F3 [1st- vs. 4th-grade, $q(3,63)=2.92$ ]. These results confirm the previously established age trend. As before, it is suggested that the phoneme boundary shifts mainly during a relatively short interval at about the age of 6 or 7 (between 6:2 and 6:8 previously; between 6:2 and $7: 4$ by the present data).

The present falling-F3 phoneme boundaries are at lower F2 starting values than in the previous study. The differences between comparable ages are too large to be due to sample variability only $(97 \mathrm{~Hz}$ for 6 -year-olds; $115 \mathrm{~Hz}$ for 9-year-olds). The 9-year-olds in the present study have even lower $F 2$ boundary values $(1369 \mathrm{~Hz})$ than did the 6-year-olds of the previous study $(1414 \mathrm{~Hz})$. The present falling-F3 stimuli differ in RMS power contour during formant transition. There are design and F2-range differences with the previous study as well. Some preliminary evidence has recently been obtained which shows that (with this kind of stimuli) the F2 phoneme boundary shifts to lower values as the power falls more rapidly during formant transitions (De Weirdt, 1987). With the present stimuli, power is indeed falling more steeply and lower F2 boundaries are obtained. So the suggestion is that the differences between the previous and the present (falling-

Table 1

Mean Phoneme-Boundary Values Across Ages (Columns) and 13 Conditions (Rows)

\begin{tabular}{lccc}
\hline & \multicolumn{3}{c}{ Age Group } \\
\cline { 2 - 4 } F3 & 1st Grade & 2nd Grade & 4th Grade \\
\hline Rising & 1,405 & 1,444 & 1,470 \\
Level & 1,366 & 1,431 & 1,432 \\
Falling & 1,317 & 1,365 & 1,369 \\
\hline
\end{tabular}


F3) phoneme boundaries are probably due to the different power contours during formant transitions.

At present, only general explanations for the agedependent phoneme-boundary change may be considered. Anatomically based changes in production characteristics may be crucial. Alternatively, the effect may relate to the maturation of the auditory peripheral and/or central nervous system. Mentioned in the introduction are the Irwin et al. (1985) results, from which the hypothesis that higher frequency information is more important in younger children's speech categorization was derived. But the resulting prediction of larger F3 influence in younger children's [pa]-[to] labeling failed. Still, the present results may be related to those of Irwin et al. (1985) at some other level. Furthermore, there might be a development in speechspecific processing. At ages 6 to 7, children learn to read. A side effect of reading mastery might be the emergence of clear representations for phonemes in isolation (cf. Bertelson, Morais, Alegria, \& Content, 1985). Reading mastery might also enhance speech perception, with visual (graphemic) information being added to the incoming speech signal. It is, of course, unclear how such events would result in phoneme-boundary changes. But it must be noted that a relationship between reading and the [pe][to] boundary has been established (De Weirdt, 1986). At constant age levels within the first grade, the [pə]-[to] phoneme boundary of poor readers is at higher F2 values than that of good readers (De Weirdt, 1985, 1986). This repeatedly demonstrated additional fact renders the age effect even more puzzling. The better readers perform like younger children. Since we may assume that good reading is positively related to general intellectual superiority, we may at least conclude that the phonemeboundary age effect is probably not related to intellectual functioning.

Further research is clearly needed, preferably taking into account production characteristics. It is also of interest to determine whether the effect would generalize to other place-of-articulation continua (e.g., alveolarvelar, vowel-consonant stimuli), and to other steady state vowel values and durations. Stimulus intensity is another variable clearly meriting further consideration. Stimulus intensity variation has been reported to result in phonemeboundary changes (Dorman \& Dougherty, 1981; SyrdalLasky, 1978), but no consistent picture as to the nature and the direction of the changes has yet emerged. On the other hand, it is well established that younger children need higher intensity levels to perform optimally in speech-perception experiments (Elliott, Longinotti, Clifton, \& Meyer, 1981). Some link may exist between the facts mentioned above and the age-dependent phoneme-boundary changes.

\section{REFERENCES}

Baker, R. J., \& Nelder, J. (1978). The GLJM System. Release 3. Generalized linear interactive modeling [Computer program manual]. Harpenden, England: Rothamsted Experimental Station.

Bertelson, P., Morais, J., Alegria, J., Content, A. (1985). Phonetic analysis capacity and learning to read. Nature, 313, 73.

DE WEIRDT, W. (1985). Developmental changes in the identification of a stop consonant continuum. Psychologica Belgica, 25, 1-16.

DE WEIRDT, W. (1986). Speech perception and frequency discrimination in good and poor readers. Manuscript submitted for publication.

DE WeIRDT, W. (1987). Perceptual effects of power contour variation during formant transitions. Manuscript in preparation.

Dorman, M. F., Dougherty, K. (1981). Shifts in phonetic identification with changes in signal presentation level. Journal of the Acoustical Society of America, 69, 1439-1440.

Elliott, L. L., Longinotti, C., Clifton, L., Meyer, D. (1981). Detection and identification thresholds for consonant-vowel syllables. Perception \& Psychophysics, 30, 411-416.

Elliott, L. L., Longinoti, C., MeYer, D., RAZ, I., \& ZuCKer, K. (1981). Developmental differences in identifying and discriminating $\mathrm{CV}$ syllables. Journal of the Acoustical Society of America, 70, 669-677.

GovaerTs, G. (1978). Perceptuele structuren van synthetische en natuurlijke klinkers. [Perceptual structure of synthetic and natural vowels]. Psychologica Belgica, 18, 27-67.

IRWIN, R. J., BAll, A. K. R., Kay, N., Stillmann, J. A., \& Rosser, J. (1985). The development of auditory temporal acuity in children. Child Development, 56, 614-620.

Simon, C., FourCin, A. J. (1978). Cross-language study of speechpattern learning. Journal of the Acoustical Society of America, 63, 925-935.

SYRDAL-LASKY, A. (1978). Effects of intensity on the categorical perception of stop consonants and isolated second formant transitions. Perception \& Psychophysics, 23, 420-432.

VAN CoILe, B. (1985). VSY. Speech symthesis program. [Computer program]. Ghent, Belgium: Laboratory for Electronics and Metrology, University of Ghent.

\section{NOTE}

1. Only four observations for each $F 2$ value are obtained, and logit analyses may seem questionable. However, a few rule-based alternatives were tried out, and essentially the same results were obtained. Similarly, a repeated measures age $\times$ F2 $\times$ F3 analysis of variance on the number of $[p 2]$ responses revealed a significant age effect $[F(2,63)=$ $4.28, p<.025]$.

(Manuscript received February 22, 1986; revision accepted for publication January 27, 1987.) 